\title{
Corrigendum: The Influence of Menstrual Cycle and Androstadienone on Female Stress Reactions: An fMRI Study
}

\author{
Ka Chun Chung ${ }^{1 *}$, Felix Peisen ${ }^{1}$, Lydia Kogler ${ }^{1,2,3}$, Sina Radke ${ }^{1,2}$, Bruce Turetsky ${ }^{4}$, \\ Jessica Freiherr ${ }^{5,6}$ and Birgit Dernt ${ }^{1,2,3,7 *}$ \\ ${ }^{1}$ Department of Psychiatry, Psychotherapy and Psychosomatics, Medical Faculty, RWTH Aachen University, Aachen, \\ Germany, ${ }^{2}$ Jülich Aachen Research Alliance - Translational Brain Medicine, Aachen, Germany, ${ }^{3}$ Department of Psychiatry \\ and Psychotherapy, Medical Faculty, University of Tübingen, Tübingen, Germany, ${ }^{4}$ Neuropsychiatry Division, Department of \\ Psychiatry, University of Pennsylvania, Philadelphia, PA, USA, ${ }^{5}$ Diagnostic and Interventional Neuroradiology, Medical Faculty, \\ RWTH Aachen University, Aachen, Germany, ${ }^{6}$ Fraunhofer Institute for Process Engineering and Packaging IW, Freising, \\ Germany, ${ }^{7}$ Institute for Neuroscience and Medicine (INM-1), Research Center Juelich, Juelich, Germany
}

Keywords: menstrual cycle, stress, social threat, cortisol, hippocampus, amygdala

\section{A Corrigendum on}

The Influence of Menstrual Cycle and Androstadienone on Female Stress Reactions: An fMRI Study

by Chung, K. C., Peisen, F., Kogler, L., Radke, S., Turetsky, B., Freiherr, J., et al. (2016). Front. Hum. Neurosci. 10:44. doi: 10.3389/fnhum.2016.00044

\section{OPEN ACCESS}

Edited and reviewed by:

Charlotte A. Boettiger,

University of North Carolina,

Chapel Hill, USA

*Correspondence:

Ka Chun Chung

kchung@ukaachen.de;

Birgit Dernt

birgit.dernt@@med.uni-tuebingen.de

Received: 20 May 2016

Accepted: 01 June 2016

Published: 21 June 2016

Citation:

Chung KC, Peisen F, Kogler L, Radke S, Turetsky B, Freiherr $J$ and Derntl B (2016) Corrigendum: The Influence of Menstrual Cycle and Androstadienone on Female Stress

Reactions: An fMRI Study.

Front. Hum. Neurosci. 10:293.

doi: 10.3389/fnhum.2016.00293
Corrigendum on: Author Contributions

In the original article, the use of "prepared the manuscript" and "supervised data collection as well as preparation of the manuscript" in the Author Contributions Statement was unintentionally vague and potentially confusing. The authors apologize for the lack of clarity. This change does not influence the scientific conclusions of the article in any way. The full corrected Author Contributions Statement appears below.

KC collected the data, performed data analyses, and wrote the manuscript. FP helped with data collection and processed the skin conductance data. LK, SR, and BT helped with data interpretation and reviewed the manuscript. JF and BD designed the study and supervised data collection as well as reviewed the manuscript.

\section{FUNDING}

The work in this manuscript was supported by the Medical Faculty, RWTH Aachen, Aachen, Germany (START 691302) and the Deutsche Forschungsgemeinschaft (DFG, IRTG 1328). BD was further supported by the Austrian Science Fund (FWF P23533).

Conflict of Interest Statement: The authors declare that the research was conducted in the absence of any commercial or financial relationships that could be construed as a potential conflict of interest.

Copyright () 2016 Chung, Peisen, Kogler, Radke, Turetsky, Freiherr and Derntl. This is an open-access article distributed under the terms of the Creative Commons Attribution License (CC BY). The use, distribution or reproduction in other forums is permitted, provided the original author(s) or licensor are credited and that the original publication in this journal is cited, in accordance with accepted academic practice. No use, distribution or reproduction is permitted which does not comply with these terms. 